\title{
A SIMPLIFIED TREATMENT OF GRAVITATIONAL INTERACTION ON GALACTIC SCALES
}

\author{
Sascha Trippe \\ Department of Physics and Astronomy, Seoul National University, Seoul 151-742, Korea \\ E-mail : trippe@astro.snu.ac.kr \\ (Received November 16, 2012; Revised December 20, 2012; Accepted January 17, 2013)
}

\begin{abstract}
I present a simple scheme for the treatment of gravitational interactions on galactic scales. In analogy with known mechanisms of quantum field theory, I assume ad hoc that gravitation is mediated by virtual exchange particles - gravitons - with very small but non-zero masses. The resulting density and mass profiles are proportional to the mass of the gravitating body. The mass profile scales with the centripetal acceleration experienced by a test particle orbiting the central mass, but this comes at the cost of postulating a universal characteristic acceleration $a_{0} \approx 4.3 \times 10^{-12} \mathrm{~m} \mathrm{sec}^{-2}$ (or $\left.8 \pi a_{0} \approx 1.1 \times 10^{-10} \mathrm{~m} \mathrm{sec}^{-2}\right)$. The scheme predicts the asymptotic flattening of galactic rotation curves, the Tully-Fisher/Faber-Jackson relations, the mass discrepancy-acceleration relation of galaxies, the surface brightness-acceleration relation of galaxies, the kinematics of galaxy clusters, and "Renzo's rule" correctly; additional (dark) mass components are not required. Given that it is based on various ad-hoc assumptions and given further limitations, the scheme I present is not yet a consistent theory of gravitation; rather, it is a "toy model" providing a convenient scaling law that simplifies the description of gravity on galactic scales.
\end{abstract}

Key words : Gravitation - Galaxies: kinematics and dynamics

\section{INTRODUCTION}

It is well-known that the treatment of gravitational interaction on galactic scales comes with a serious flaw denoted as the missing mass problem. Since the seminal works by Zwicky (1933) and Rubin et al. (1980), it has become clear that the dynamical mass necessary to explain the kinematics of galactic systems exceeds the observed luminous mass by up to one order of magnitude. On the one hand, this observation has lead to the postulate of non-luminous and non-baryonic dark matter that accounts for the observed discrepancy (Ostriker \& Peebles 1973), eventually evolving into the modern $\Lambda$ CDM standard model of cosmology (e.g., Bahcall et al. 1999). On the other hand, various proposals for modified laws of inertia and/or gravity have been made to address the missing mass problem, also in view of open questions regarding standard cosmology (e.g., Kroupa 2012); the most successful candidate to date appears to be "Milgrom's law" of modified inertia (Milgrom 1983; Famaey \& McGaugh 2012).

To date, a fully satisfactory description of gravitational interaction on galactic scales has not been found. Dark matter models are struggling with "finetuning problems" regarding the distribution of dark matter within and around galaxies (e.g., Kroupa 2012), whereas "Milgrom's law" is based on an entirely empirical ad-hoc modification of Newton's law of inertia (e.g., Famaey \& McGaugh 2012).

My work aims at a simple, convenient, physically motivated description of gravity on galactic scales. In analogy with the well-established concepts of particle physics and quantum field theories (see e.g., Griffith 2008 for an overview), I assume ad hoc that gravitational interactions between masses are mediated by discrete virtual particles dubbed gravitons. Assuming further that these gravitons have non-zero mass and obey certain rules of interaction, the resulting total mass distributions (composed of source masses plus graviton distributions) can be treated by standard Newtonian dynamics (see e.g., Binney \& Tremaine 2008 for a thorough review of stellar and galactic dynamics). The resulting scaling relations for mass profiles and rotation curves agree satisfactorily with observations.

\section{GRAVITATIONAL INTERACTION}

\subsection{Fundamental Assumptions}

In order to provide a scheme of gravitational interaction, I start with the following fundamental assumptions:

A1. Gravitational interactions are mediated by discrete particles, gravitons.

A2. Gravitons are virtual particles arising from quantum fluctuations - the mechanism commonly employed in quantum field theories.

A3. Gravitons have a non-zero mass. 
A4. Interactions may only occur (1) between two real masses; (2) between a real mass and a graviton emitted by a real mass.

\subsection{Consequences}

Assumptions A1-4 lead to several consequences which can be partially tested by observations. Unless stated otherwise, the terms "gravitational system" or "dynamical system" denote a system composed of a pointlike, real, baryonic, luminous source mass $M_{0}$ and a quasi-massless test particle orbiting $M_{0}$ on a circular orbit at radial distance $r$ with circular speed $v_{\mathrm{c}}$.

\subsubsection{Gravitons Are "Dark"}

Postulating gravitons as mediators of gravitational interactions (assumption A1) implies that they couple to mass, with mass playing the role of the "charge" of gravity. Gravitons should not be expected to take part in electromagnetic/electroweak or strong nuclear interactions (see e.g., Griffith 2008 for an overview). Accordingly, gravitons do not interact with photons electromagnetically, they are "dark" in the sense of being "invisible" to electromagnetic radiation.

\subsubsection{Graviton Masses}

The creation of virtual massive particles from quantum fluctuations (assumptions A2 and A3) is, a priori, a violation of the principle of conservation of energy. This violation is only possible as long as it is "temporary" within the limits of Heisenberg's uncertainty relation between uncertainties in energy and time. In our case,

$$
\Delta E \Delta t \approx \hbar
$$

where $\Delta E=m_{g} c^{2}$ is the energy of a graviton with mass $m_{g}, c$ is the speed of light, $\Delta t$ is the lifetime of the virtual particle, and $\hbar=1.055 \times 10^{-34} \mathrm{Js}_{\mathrm{s}}$ is the reduced form of Planck's constant (e.g., Griffith 2008).

A range of gravitational interactions on cosmological scales requires graviton lifetimes on the order of the Hubble time, meaning $\Delta t \gtrsim 1.4 \times 10^{10}$ yrs. Accordingly, we require

$$
m_{g}=\frac{\Delta E}{c^{2}} \lesssim 3 \times 10^{-69} \mathrm{~kg} \approx 1.5 \times 10^{-33} \mathrm{eV} c^{-2}
$$

This value is about 40 orders of magnitude smaller than the masses of virtual exchange particles occurring in particle physics - a necessary consequence of the vast difference in size scales (see also Goldhaber \& Nieto 2010 for a recent review of graviton mass limits).

\subsubsection{Density and Mass Profiles}

I assume in the following that the source mass $M_{0}$ radiates away gravitons without losing mass itself; this is a corollary of assumption A2. Consistency with the classical theory of gravitational fields requires that (1) the graviton density profile scales linearly with $M_{0}$, and (2) the density profile obeys the inverse-square law with respect to the distance in gravitational fields (and radiation fields in general). Accordingly, we find

$$
\rho=M_{0} \beta r^{-2}
$$

where $\rho$ is the mass density of the graviton distribution, $r$ is the radial distance from $M_{0}$, and $\beta$ is a scaling parameter of the dimension of an inverse length.

The mass profile is found from integration of $\rho(r)$. Demanding that $\beta$ does not depend on $r$ explicitly, ${ }^{*}$ we find the extra mass enclosed within a distance $r$ to be

$$
M_{\mathrm{ex}}=4 \pi M_{0} \beta r
$$

whereas the total enclosed mass is given by

$$
M_{\mathrm{tot}}=M_{0}+M_{\mathrm{ex}}=M_{0}(1+4 \pi \beta r) \text {. }
$$

\subsubsection{Enclosed Mass vs. Centripetal Acceleration}

To permit a comparison of arbitrary gravitational systems, we need to bring the scaling parameter $\beta$ in Eq. 4 into a form that (1) provides a characteristic scale for a given dynamical system, and (2) does not depend on the coordinate $r$ explicitly. Both conditions are fulfilled by choosing

$$
\beta=\frac{2 a_{0}}{v_{\mathrm{c}}^{2}}
$$

where $v_{\mathrm{c}}^{2} / 2$ is the kinetic energy per unit mass of a test particle orbiting $M_{0}$ with circular speed $v_{\mathrm{c}}$, and $a_{0}$ is a constant of the dimension of an acceleration. Conveniently, the kinetic energy is scale-free, i.e., invariant under simultaneous scaling of coordinates and time by factors $x \neq 0$. Inserting $\beta$ into Eqs. 4 and 5 leads to a new expression for the enclosed total mass,

$$
M_{\mathrm{tot}}=M_{0}\left(1+8 \pi \frac{a_{0}}{a_{\mathrm{c}}}\right)
$$

with $a_{\mathrm{c}}=v_{\mathrm{c}}^{2} / r$ being the centripetal acceleration experienced by a test particle orbiting $M_{0}$ with circular speed $v_{\mathrm{c}}$ at radial distance $r$.

\subsubsection{Absence of Self-Interaction}

Assumption A4 is crucial for the self-consistency of the "graviton picture" of gravitation I use. Under assumptions A1 and A3, gravity is mediated by gravitons that have mass themselves. As mass can be regarded as the "charge" of gravitation, we may, a priori, expect interactions also between virtual masses, leading to the

* Meaning here specifically: $\beta$ may be a function of a given welldefined distance $r^{\prime}$ but not of the coordinate $r$ in general. This permits integration of $\rho(r)$ over $r$ from 0 to $r^{\prime}$ and a subsequent substitution $r^{\prime} \longrightarrow r$. 
creation of additional masses, which interact again, and so on. Those interactions of higher order can result in a divergence in the creation of mediator particles, leading to massive modifications of the overall interactions - especially to violations of the $r^{-2}$ law of gravity (and radiation in general). This is a situation commonly encountered and treated in quantum chromodynamics, expressed especially in the confinement of quarks (Griffith 2008).

Assuming a quasi-massless test particle interacting with $M_{0}$, assumption A4 prevents those divergences:

- By permitting interactions between real masses, we ensure that the test mass "sees" the source mass $M_{0}$ in the usual Newtonian way.

- By permitting interactions between real masses and virtual masses emitted by a real mass, we ensure that the test mass "sees" the distribution of primary gravitons emitted by $M_{0}$.

- By preventing any other interaction, we suppress interactions of higher order, potentially leading to divergences and/or confinements.

As mentioned above, the conditions imposed by assumption A4 are far from obvious. In quantum field theories, those conditions are usually traced back to conserved quantities that impose selection rules for allowed interactions; prominent examples are spins or the color charges of quantum chromodynamics (Griffith 2008).

\section{TESTS}

\subsection{Galactic Rotation Curves}

The circular speed of a test particle orbiting $M_{0}$ is related to Eq. 7 by

$$
v_{\mathrm{c}}^{2}=\frac{G M_{\mathrm{tot}}}{r}=\frac{G M_{0}}{r}\left(1+8 \pi \frac{a_{0}}{a_{\mathrm{c}}}\right)
$$

with $G$ denoting Newton's constant. As long as $8 \pi a_{0} / a_{\mathrm{c}} \ll 1$, the first summand in the bracket dominates, resulting in (quasi-)Keplerian motion. However, in the limit $8 \pi a_{0} / a_{\mathrm{c}} \gg 1$, the second summand dominates and we have

$$
v_{\mathrm{c}}^{2} \approx \frac{8 \pi G M_{0}}{r} \times \frac{a_{0}}{a_{\mathrm{c}}} .
$$

Exploiting the fact that $a_{\mathrm{c}}=v_{\mathrm{c}}^{2} / r$, we can rewrite Eq. 9 as

$$
v_{\mathrm{c}}{ }^{4} \approx 8 \pi G M_{0} a_{0}=\text { const. }
$$

Accordingly, one finds that, in the limit of strong centripetal accelerations, the circular speed is Keplerian; it approaches a constant value when approaching the limit of weak centripetal accelerations. This is in agreement with the observed behavior of galactic rotation curves (Rubin et al. 1980).

\subsection{Tully-Fisher/Faber-Jackson Relations}

Next to the fact that rotation curves flatten out asymptotically, Eq. 10 provides additional information: it states that, asymptotically,

$$
v_{\mathrm{c}}{ }^{4} \propto M_{0} \text {. }
$$

This is in agreement with the empirical Tully-Fisher relation between rotation speeds of spiral galaxies and their luminous masses (Tully \& Fisher 1977). A relation as predicted by Eq. 10 is observationally established over ten orders of magnitude of mass for gravitationally bound systems, from dwarf galaxies to galaxy clusters (cf., Fig. 48 of Famaey \& McGaugh 2012). Likewise, Eq. 10 provides an explanation for the empirical Faber-Jackson relation between the luminosity - which is proportional to the source mass in my interaction scheme - of elliptical galaxies and their velocity dispersions (Faber \& Jackson 1976).

\subsection{Mass Discrepancy-Acceleration Relation}

Eq. 7 links the total enclosed mass, the source mass, and the centripetal acceleration experienced by a test particle orbiting the source mass. By rewriting this equation slightly, we can formulate a mass discrepancyacceleration relation (McGaugh 2004) that links the mass discrepancy, defined as the ratio $M_{\text {tot }} / M_{0}$, and acceleration directly:

$$
\frac{M_{\mathrm{tot}}}{M_{0}}=1+8 \pi \frac{a_{0}}{a_{\mathrm{c}}} .
$$

Relation 12 is illustrated in Fig. 1a for a range of weak centripetal accelerations $a_{\mathrm{c}}$. In addition, Fig. $1 \mathrm{~b}$ shows the mass discrepancy as function of the Newtonian gravitational acceleration expected if $M_{0}$ were equal to the total enclosed mass, $g_{N} \equiv a_{\mathrm{c}} /\left(M_{\mathrm{tot}} / M_{0}\right)$. Comparison to Fig. 10 of Famaey \& McGaugh (2012) indicates a satisfactory agreement between Eq. 12 and observations for $a_{0}=4.3 \times 10^{-12} \mathrm{~m} \mathrm{sec}^{-2}( \pm 5 \%)$.

\subsection{Surface Brightness vs. Acceleration}

In the limit of weak centripetal accelerations, we can use Eq. 10 and rewrite it as

$$
\frac{v_{\mathrm{c}}^{4}}{r^{2}} \approx 8 \pi G a_{0} \frac{M_{0}}{r^{2}}
$$

For a sufficiently flat stellar system, we can define a (luminous) surface density $\Sigma \propto M_{0} / r^{2}$. Inserting $\Sigma$ into Eq. 13 and exploiting the fact that $a_{\mathrm{c}}=v_{\mathrm{c}}{ }^{2} / r$, we may conclude that

$$
a_{\mathrm{c}}^{2} \propto \Sigma \quad \text { or } \quad a_{\mathrm{c}} \propto \Sigma^{1 / 2}
$$

Such a correlation is indeed observed (see Fig. 9 of Famaey \& McGaugh 2012). 

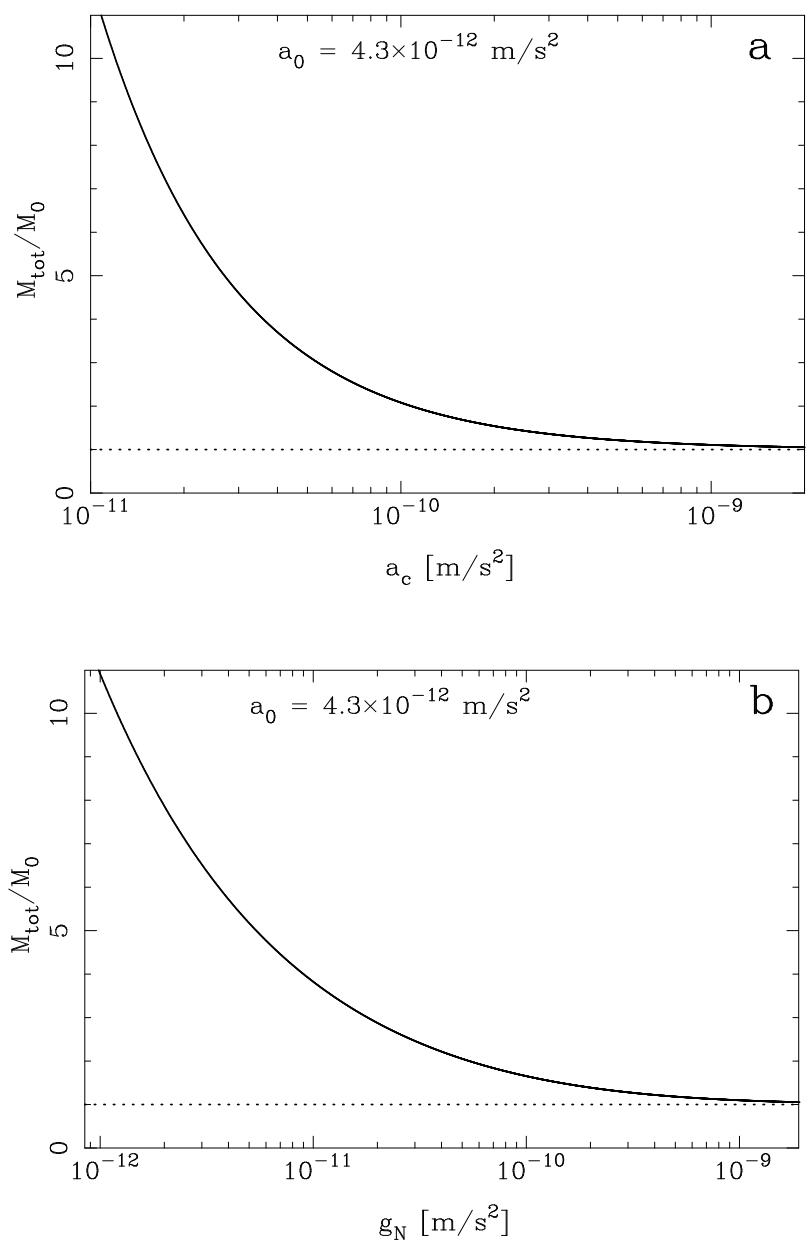

Fig. 1. - Theoretical mass discrepancy-acceleration relations. a. The ratio $M_{\text {tot }} / M_{0}$ as function of centripetal acceleration $a_{\mathrm{c}}$ according to Eq. 12 . b. $M_{\mathrm{tot}} / M_{0}$ as function of the Newtonian gravitational acceleration expected if $M_{0}$ were the total mass, $g_{N} \equiv a_{\mathrm{c}} /\left(M_{\mathrm{tot}} / M_{0}\right)$. The characteristic acceleration used here is $a_{0}=4.3 \times 10^{-12} \mathrm{~m} \mathrm{sec}^{-2}$. These diagrams should be compared to Fig. 10 of Famaey \& McGaugh (2012).

\subsection{Galaxy Clusters}

The interaction scheme I use is not restricted to galaxies alone but also becomes visible in the dynamics of galaxy clusters, likewise leading to observable mass discrepancies. Unfortunately, application of Eq. 7 is not straightforward because galaxy clusters are not pointlike masses but extended mass distributions with most of their masses apparently stored in the X-ray luminous intra-cluster medium (e.g., Sparke \& Gallagher 2007).

I estimate the mass discrepancy crudely - accurate to factors on the order of a few - by using the velocity dispersions and core radii of the clusters as proxies for $v_{\mathrm{c}}$ and orbit radii, respectively. For the four clusters Virgo, Coma, Perseus, and RDCS 1252.9-2927 (from
Table 7.1 of Sparke \& Gallagher 2007), this leads to theoretical mass discrepancies of about 2 to 4 ; the observed values are about 6 to 10 . Given the crudeness of the estimate, I conclude that theoretical and observational values are consistent with each other; especially, as both are of the same order of magnitude $\lesssim 10$.

\section{6 "Renzo's Rule"}

Observationally, dynamical and (luminous) baryonic surface densities in disk galaxies are closely related, expressed - among others - by "Renzo's rule" (Sancisi 2004) which states that "for any feature in the luminosity profile, there is a corresponding feature in the rotation curve". This empirical rule has been used as an argument against the presence of dark matter distributions in disk galaxies because these are, a priori, independent from structures in the distributions of luminous mass (Famaey \& McGaugh 2012).

In the frame of the interaction scheme presented in this work, "Renzo's rule" is self-evident due to the unique relation between (luminous) source mass and total enclosed mass (Eqs. 7, 8).

\section{DISCUSSION}

Starting from the ad-hoc assumptions A1-4, I arrive at a simple description of gravitational interaction on galactic scales. This "graviton picture" of gravity makes several predictions about galactic dynamics that are consistent with observations. Compared to standard models of galactic dynamics, the scheme I propose seems preferable because it removes the need for a separate "dark mass" component - thus providing a simplified description. Notably, the "graviton picture" seems to be the only model to date that predicts the mass discrepancy-acceleration relation (Section 3.3, Fig. 1), and thus the transitional regime between Newtonian and modified gravitational interaction, correctly - modified laws of gravity/inertia or dark matter models in general make predictions on the asymptotic behavior of stellar systems only.

Although it seems that my work is the first to explicitly apply the assumption of massive gravitons to galactic dynamics, the concept of massive gravitons or "massive gravity" - itself has been discussed in field theories for more than seven decades (see, e.g., Goldhaber \& Nieto 2010; Hinterbichler 2012 for reviews) and has been applied to various aspects of cosmology (e.g., Cardone, Radicella \& Parisi 2012).

Even though my interaction scheme appears successful on galactic scales, it is obviously incomplete. It is formulated in the frame of classical Newtonian dynamics, meaning it is intrinsically non-relativistic. This implies that the scheme breaks down at small scales where relativistic deviations from Newtonian dynamics become noticeable - on scales of the size of the solar system and less - as well as at large scales where cosmological effects, like the Hubble flow, become evident 
- on scales of mega-parsecs and beyond.

A further peculiarity of the description I provide is encoded in the formulation of the scaling parameter $\beta$ in Eq. $6 .^{\dagger}$ The choice I make is, to a certain extent, technically self-evident: Eq. 6 provides a characteristic kinematic size scale, is independent of the radial coordinate, and is based on the scaleinvariant, conserved kinetic energy. However, the apparent astrophysical consequences discussed throughout Section 3 enforce a remarkable postulate: there is a characteristic acceleration $a_{0} \approx 4.3 \times 10^{-12} \mathrm{~m} \mathrm{sec}^{-2}$ (or $8 \pi a_{0} \approx 1.1 \times 10^{-10} \mathrm{~m} \mathrm{sec}^{-2}$ ) which is a universal constant of nature. The origin of this characteristic acceleration remains unclear; ${ }^{\ddagger}$ I note however that it is well-known empirically (cf., e.g., Famaey \& McGaugh 2012, and references therein).

Given that the interaction scheme I propose is based on various ad-hoc assumptions, and given its limitations discussed above, it is clear that the "graviton picture" is not yet a consistent theory of gravitation. Instead, it should be seen as a "toy model" that provides a simple scaling law for gravitational interaction on galactic scales and that is simpler than standard models (which require additional mass components). This seems valuable especially in view of modern numerical simulations exploring galactic to cosmological scales (e.g., Kim et al. 2011).

Despite the various limitations stated above, one can think of several observational signatures that could be used to further probe the "graviton picture", in addition to the "usual suspects" discussed throughout Section 3:

Dynamical friction. To a large extent, the dynamics of stellar systems is governed by dynamical friction (cf. Ch. 8.1 of Binney \& Tremaine 2008). The assumption of dark matter halos around galaxies predicts a variety of dynamical signatures ranging from the evolution of binary star orbits (e.g., Hernandez \& Lee 2008) to the effects of galaxy encounters (e.g., Dubinski, Mihos \& Hernquist 1999); accordingly, models for dark matter and/or modified laws of gravity can, and have been, examined by comparing the results of N-body simulations with observations of those systems. The "graviton picture" makes a strong prediction: as a corollary of assumption A4, only baryonic masses experience dynamical friction in a "graviton halo"; the graviton distributions themselves do not interact. This is sharply distinct from the case of dark matter halos where both baryonic and dark mass particles are supposed to ex-

$\dagger \mathrm{I}$ also note an analogy provided by atomic physics. Rydberg's constant $R_{\infty}$ is the only constant of nature of the dimension of an inverse length (e.g., Lang 2006). In Bohr's model, $R_{\infty}$ is the inverse wavelength of a photon with the energy of the ground state of a hydrogen atom. This means that $R_{\infty}$ actually provides an energy scale; it is only a constant of nature because all hydrogen atoms are identical. Likewise, $\beta$ provides a characteristic energy scale for dynamical systems.

¥ Though this discussion might be loosely related to the discussion of Mach's principle; see e.g., Bondi \& Samuel (1997). perience dynamical friction. Likewise, modified laws of gravity do not introduce any extra masses, meaning they do not predict dynamical friction beyond the one due to luminous mass. Accordingly, observational evidence, e.g., in galaxy collisions, for interactions corresponding to dynamical friction of baryonic mass in "dark halos" without interaction among those halos would provide support for the "graviton picture".

Collisions of galaxy clusters. The "graviton picture" demands a direct coupling of a luminous baryonic source mass and the extra mass due to the surrounding graviton distribution (cf. Eqs. 4, 5, 7). In the standard dark matter picture, luminous and dark mass may be distributed independently. A test of these scenarios is provided by colliding clusters of galaxies: when the cluster cores pass through each other, the hot, $\mathrm{X}$-ray bright intra-cluster gas, which comprises a large fraction of the baryonic cluster masses, experiences collisional ram-pressure and lags behind the collisionless galaxies and dark matter halos - if those actually exist. Accordingly, a comparison of the spatial distributions of total mass - derived from gravitational lensing of background sources - and luminous mass is able to test dark matter and/or modified gravity models. An important example is provided by the "Train-Wreck Cluster" A520 (Markevitch et al. 2005). In A520, observations find that the spatial distributions of total and luminous mass coincide (Jee et al. 2012) - in agreement with the "graviton picture". Another example is provided by the "Bullet Cluster" 1E0657-56 (Barrena et al. 2002). Here the situation is more complicated: on the one hand, observations indicate a spatial separation of dark and luminous mass; on the other hand, the cluster kinematics is found to be inconsistent with $\Lambda$ CDM cosmology (Lee \& Komatsu 2010).

Gravitational lensing. The "graviton picture" makes a specific prediction for the distribution of the nonluminous extra mass around a luminous source mass: as it assumes that gravity is the result of a radiation composed of massive particles, the density distribution of these particles has to obey the usual $r^{-2}$ law of radiation (cf. Eq. 3). This prediction has been tested by gravitational lensing studies that probe the density profiles of galaxies; observational results are in agreement with $\rho \propto r^{-2}$ as demanded by the "graviton picture" (Gavazzi et al. 2007).

Cosmology. A non-zero mass implies a limited life time of gravitons (see Section 2.2.2). Accordingly, a decay of gravitons on cosmological time scales should modify the cosmic expansion history; especially, an acceleration corresponding to the one commonly ascribed to dark energy (Bahcall et al. 1999) might occur. Recent studies (Cardone, Radicella \& Parisi 2012) indeed conclude that massive gravity is able to reproduce the observed cosmological parameters.

Evidently, further theoretical as well as observational work is required to understand the limitations and implications of the interaction scheme I present in 
this study. Eventually, the "graviton picture" might play a role for galactic dynamics which is analogous to the one played by Bohr's "planetary system" model of the hydrogen atom for atomic physics: even though it is an over-simplified, coarse approximation of the actual physical situation, it provides a successful and convenient quantitative description for a wide range of phenomena. Ironically, the "graviton picture" resolves the ambiguity "dark matter or modified gravity" by assuming "dark matter through modified gravity".

\section{CONCLUSIONS}

Based on a set of four ad-hoc assumptions, I present a simple scheme for the treatment of gravitational interaction on galactic scales:

1. I assume that gravitation is mediated by gravitons, which are virtual, discrete exchange particles with non-zero mass. These gravitons ought to interact gravitationally but not electromagnetically, meaning they are "invisible" to light.

2. Demanding consistency with classical field theory, the mass and density profiles of the graviton distribution around a source mass are proportional to the mass of the source. The density profile obeys the usual inverse-square-of-distance law of gravitational fields.

3. The mass profile contains a scaling factor $\beta$ that can be written as the ratio of a universal, constant acceleration and the kinetic energy per unit mass of a test particle orbiting the source mass. Empirically, the characteristic acceleration is $a_{0} \approx$ $4.3 \times 10^{-12} \mathrm{~m} \mathrm{sec}^{-2}$. In the chosen formulation, the mass profile is a function of the centripetal acceleration experienced by a test particle orbiting the source mass.

4. Mass profiles and resulting rotation curves predict the asymptotic flattening of galactic rotation curves, the Tully-Fisher/Faber-Jackson relations, the mass discrepancy-acceleration relation of galaxies, the surface brightness-acceleration relation of galaxies, the kinematics of galaxy clusters, and "Renzo's rule" correctly. Additional future observational tests could be provided by dynamical friction in stellar systems, collisions of galaxy clusters, gravitational lensing by galaxies, and the evolution of cosmic expansion.

5 . The interaction scheme is non-relativistic, meaning it should break down on small - solar system - as well as cosmological scales.

Within its known limitations, the "graviton picture" provides a useful tool for studies of galactic dynamics. Obviously, its range of validity needs to be explored more carefully in future observational as well as theoretical/numerical studies.

\section{ACKNOWLEDGMENTS}

I am grateful to Junghwan OH, TAESEOK LeE, JAEYoung KIM, and JONG-Ho PARK (all at SNU) for valuable technical support. This work made use of the software package DPUSER ${ }^{\S}$ developed and maintained by ThOMAS OтT at MPE Garching. I acknowledge financial support from the Korean National Research Foundation (NRF) via Basic Research Grant 2012R1A1A2041387. Last but not least, I am grateful to an anonymous reviewer whose careful report helped to improve this paper.

\section{REFERENCES}

Bahcall, N. A., Ostriker, J. P., Perlmutter, S., \& Steinhardt, P. J. 1999, The Cosmic Triangle: Revisiting the State of the Universe, Science, 284, 1481

Barrena, R., Biviano, A., Ramella, M., Falco, E. E., \& Seitz, S. 2002, The Dynamical Status of the Cluster of Galaxies 1E0657-56, A\&A, 386, 816

Binney, J., \& Tremaine, S. 2008, Galactic Dynamics, 2nd edn. (Princeton: Princeton University Press)

Bondi, H., \& Samuel, J. 1997, The Lense-Thirring Effect and Mach's Principle, Phys. Letters A, 228, 121

Cardone, V. F., Radicella, N., \& Parisi, L. 2012, Constraining Massive Gravity with Recent Cosmological Data, Phys. Mod. Rev. D, 85, 124005

Dubinski, J., Mihos, J. C., \& Hernquist, L. 1999, Constraining Dark Halo Potentials with Tidal Tails, ApJ, 526, 607

Faber, S. M., \& Jackson, R. E. 1976, Velocity Dispersions and Mass-to-Light Ratios for Elliptical Galaxies, ApJ, 204,668

Famaey, B., \& McGaugh, S. S. 2012, Modified Newtonian Dynamics (MOND): Observational Phenomenology and Relativistic Extensions, Living Rev. Relativity, 15, 10

Gavazzi, R., Treu, T., Rhodes, J. D., et al. 2007, The Sloan Lens ACS Survey. IV. The Mass Density Profile of EarlyType Galaxies out to 100 Effective Radii, ApJ, 667, 176

Goldhaber, A. S., \& Nieto, M. M. 2010, Photon and Graviton Mass Limits, Rev. Mod. Phys., 82, 939

Griffith, D. 2008, Introduction to Elementary Particles (Weinheim: Wiley-VCH)

Hernandez, X., \& Lee, W. H. 2008, The Tightening of Wide Binaries in dSph Galaxies through Dynamical Friction as a Test of the Dark Matter Hypothesis, MNRAS, 387, 1727

Hinterbichler, K. 2012, Theoretical Aspects of Massive Gravity, Rev. Mod. Phys., 84, 671

Jee, M. J., Mahdavi, A., Hoekstra, H., et al. 2012, A Study of the Dark Core in A520 with the Hubble Space Telescope: The Mystery Deepens, ApJ, 747, 96

Kim, J.-H., Park, C., Rossi, G., Lee, S. M., \& Gott, J. R. III. 2011, The New Horizon Run Cosmological N-Body Simulations, JKAS, 44, 217

Kroupa, P. 2012, The Dark Matter Crisis: Falsification of the Current Standard Model of Cosmology, PASA, 29, 395

\footnotetext{
$\S_{\text {http: }} / /$ www.mpe.mpg.de/ ott/dpuser/dpuser.html
} 
Lang, K. R. 2006, Astrophysical Formulae. Vol. I, 3rd edn. (Berlin: Springer)

Lee, J., \& Komatsu, E. 2010, Bullet Cluster: a Challenge to $\Lambda$ CDM Cosmology, ApJ, 718, 60

Markevitch, M., Govoni, F., Brunetti, G., \& Jerius, D. 2005, Bow Shock and Radio Halo in the Merging Cluster A520, ApJ, 627, 733

McGaugh, S. S. 2004, The Mass Discrepancy-Acceleration Relation: Disk Mass and the Dark Matter Distribution, ApJ, 609, 652

Milgrom, M. 1983, A Modification of the Newtonian Dynamics as a Possible Alternative to the Hidden Mass Hypothesis, ApJ, 270, 365

Ostriker, J. P., \& Peebles, P. J. E. 1973, A Numerical Study of the Stability of Flattened Galaxies: Or, Can Cold Galaxies Survive?, ApJ, 186, 467

Rubin, U. C., Ford, W. K. Jr., \& Thonnard, N. 1980, Rotational Properties of $21 \mathrm{Sc}$ Galaxies with a Large Range of Luminosities and Radii, from NGC $4605(\mathrm{R}=4 \mathrm{kpc})$ to UGC 2885 ( $\mathrm{R}=122 \mathrm{kpc})$, ApJ, 238, 471

Sancisi, R. 2004, The Visible Matter - Dark Matter Coupling, in: Ryder, S. D., et al. (eds.), Dark Matter in Galaxies, IAU Symposium, 220, 233

Sparke, L. S., \& Gallagher, J. S. III. 2007, Galaxies in the Universe, 2nd edn. (Cambridge: Cambridge University Press)

Tully, R. B., \& Fisher, J. R. 1977, A New Method of Determining Distances to Galaxies, A\&A, 54, 661

Zwicky, F. 1933, Die Rotverschiebung von Extragalaktischen Nebeln, Helvet. Phys. Acta, 6, 110 